\title{
A Basis for Improving Numerical Forecasting in the Gulf Area by Assimilating Doppler Radar Radial Winds
}

\author{
Fathalla A. Rihan ${ }^{1}$, Chris G. Collier ${ }^{2}$ \\ ${ }^{1}$ Department of Mathematical Sciences, College of Science, United Arab Emirates University, Al-Ain, UAE \\ (Permanent address: Faculty of Sceince, Helwan University, Cairo, EGYPT) \\ ${ }^{2}$ National Center for Atmospheric Science, School of Earth and Environment, University of Leeds, Leeds, UK \\ E-mail:frihan@uaeu.ac.ae,C.G.Collier@leeds.ac.uk \\ Received June 7, 2010; revised July 1, 2010; accepted July 26, 2010
}

\begin{abstract}
An approach to assimilate Doppler radar radial winds into a high resolution Numerical Weather Prediction (NWP) model using 3D-Var system is described. We discuss the types of errors that occur in radar radial winds. Some related problems such as nonlinearity and sensitivity of the forecast to possible small errors in initial conditions, random observation errors, and the background states are also considered. The technique can be used to improve the model forecasts, in the Gulf area, at the local scale and under high aerosol (dust/sand/pollution) conditions.
\end{abstract}

Keywords: 3D-Var, Data Assimilation, Doppler Winds, Errors, NWP, Nonlinearity, Sensitivity

\section{Introduction}

Numerical Weather Prediction (NWP) is considered as an initial-boundary value problem: given an estimate of the present state of the atmosphere, the model simulates (forecasts) its evolution. Specification of proper initial conditions and boundary conditions for numerical dynamical models is essential in order to have a well-posed problem and subsequently a good forecast model (A well-posed initial/boundary problem has a unique solution that depends continuously on the initial/boundary conditions). The goal of data assimilation is to construct the best possible initial and boundary conditions, known as the analysis, from which to integrate the NWP model forward in time.

Assimilation of Doppler radar wind data into atmospheric models has recently received increasing attention due to developments in the use of limited area high resolution numerical models for weather prediction [1]. The models require observations with high spatial and temporal resolution to determine the initial conditions, for which purpose radar data are particularly appealing. However, the resolution of Doppler radar observations is much higher than that of the mesoscale NWP model. Before the assimilation, these data must be preprocessed to be representative of the characteristic scale of the model. To reduce the representativeness error and correspond the data more closely to the model resolutions than do the raw observations, one may spatially interpolate from the raw data to generate the so called super-observations; see Section 8 .

Over the last thirty years or so networks of weather radars, providing measurements of radar reflectivity, from which rainfall has been estimated, have been established within operational observing systems. Initially the radars, operating at S-band $(10 \mathrm{~cm})$ or C-band $(5-6 \mathrm{~cm})$ wavelengths did not have the capability to measure the motion of the targets (mainly hydrometeors but also insects and birds, and for high power systems, refractive index inhomogeneities) towards or away from the radar site. During the last twenty years or so weather radars having Doppler capability measuring radial motion of the targets have become standard such that now in Europe well over half of the operational radars are Doppler systems (see [2,3]). Recently, Doppler radar radial winds have been assimilated into NWP models as vertical wind profiles derived from Velocity Azimuth Display (VAD) analysis $[4,5]$, and using variational techniques [6-8].

In order to assimilate Doppler radial velocity observations, the observation errors which come from several sources are estimated for inclusion in the variational system. In this paper we outline the likely errors in estimates of Doppler radar radial winds, and how they might be represented mathematically. To illustrate the results, we apply the methodology to artificial data. We describe the radial wind and error representation as part of a system 
for generating simulated data for use in the 3D-Variational (3D-Var) system. In Section 2, we discuss the types of errors in radar radial winds. Section 3 describes a simulation model which is used to analyse actual radial winds. We outline how such observations are assimilated in 3D-Variational (3D-Var) system in Section 4. Some associated issues due to the nonlinearity are described in Section 5. A proposed methodology is described to derive the variation of the errors with range in Section 6. Direct assimilation of radial winds in PPI format, and preprocessing the data are discussed in Sections $7 \& 8$. Steps of NWP and conclusions are given in Sections $9 \&$ 10. This work is mainly based on the work described in Rihan et al. [1].

\section{Errors in Doppler Radial Velocity}

Targets moving away from or towards a radar produce a Doppler shift between the frequency of the transmitted signal (pulse), and the signal reflected from the targets and received back at the radar. However, ambiguities may arise in these measurements due to range folding and velocity aliasing [9]. Fortunately procedures have been developed to minimize these problems [10].

Other problems remain, namely the existence of data holes (where there are no targets), and irregular coverage, instrumental noise and sampling errors. Various types of interpolation schemes have been used to fill in data holes and poor coverage [11], although such schemes are unnecessary when three dimensional assimilation schemes are implemented. However, the impacts of instrumental noise and sampling are more problematic. May et al. (1989) discuss, and assess, a number of techniques used to estimate the Doppler shift in the received signals. The Doppler shift is proportional to the slope of the phase of the autocorrelation function (at zero lag) of the returned signals. An estimator of the shift is the phase at the first lag divided by the value of the lag in time units. This is known as pulse pair processing, and may be improved by averaging more than one value of the phase divided by the lag (poly pulse pair).

Sampling errors depend upon the size of the pulse volume corresponding to each data point. In practice the sampling errors could be weakly correlated from point to point, but only a very small additional error will be introduced if this is ignored. Practically, sampling errors dominate since instrumental errors are usually minimized in operational systems. In the following we outline a system for creating artificial radar radial wind data sets within which different types of error may be included. Figure 1 shows schematics of the impact upon a Gaussian Doppler spectrum of various effects of strong wind shear along the pulse volume, and instrumentally induc- ed effects. Several of these effects upon the Doppler spectrum may be present in the same radar image, and, in the case of geophysically-induced effects, their magnitude may vary with range and azimuth. The height and size of the pulse volumes will increase with increasing distance from the radar.

\section{Simulation of Doppler Radial Winds}

The construction of artificial radar data sets has been carried out for several studies over the last twenty years [12-14]. Consider a conical radar scan (see Figure 2) in a Cartesian coordinate system (x, y, z). The components of the wind field corresponding to these coordinates are $\mathrm{u}, \mathrm{v}$ and $\mathrm{w}$ respectively. It is assumed that velocity-range folding has been removed. The wind is assumed to vary with height according to an Ekman spiral with variable surface friction. The wind direction at the top of the boundary layer is parallel to the isobars, whilst the wind direction at the surface is in the direction of the lower pressure due to the surface friction, the coriolis force and pressure gradient force.

$$
\begin{aligned}
& u=-U_{g} e^{-a z} \sin a z, \\
& v=U_{g}\left(1-e^{-a z} \cos a z\right),
\end{aligned}
$$

where $U_{g}$ is the geostrophic wind, $a=\sqrt{f / 2 k}, f$ is the coriolis parameter and $\mathrm{k}$ is the eddy exchange coefficients $\left(\approx 5 \times 10^{4} \mathrm{~cm}^{2} \mathrm{sec}^{-1}\right)$ in middle latitudes.

The simulated data are assumed to be available on the measurement points (see Figure 2). The radial velocity is calculated from

$$
v_{r}=u \sin \theta \cos \vartheta+v \cos \theta \cos \vartheta+w \sin \vartheta
$$

where $u, v, w$ are the wind components; and $\theta, \vartheta$ are the azimuth angle, and the elevation angle of the radar beam.
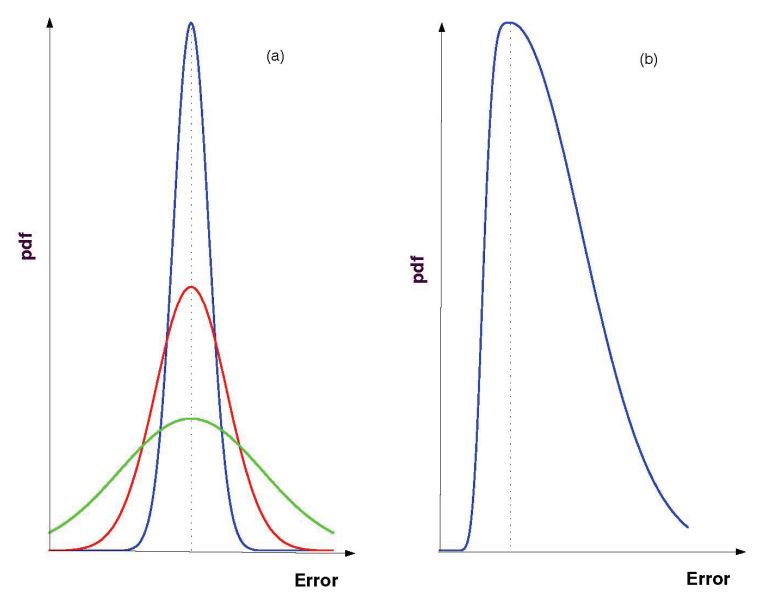

Figure 1: Distribution of wind speed error. 

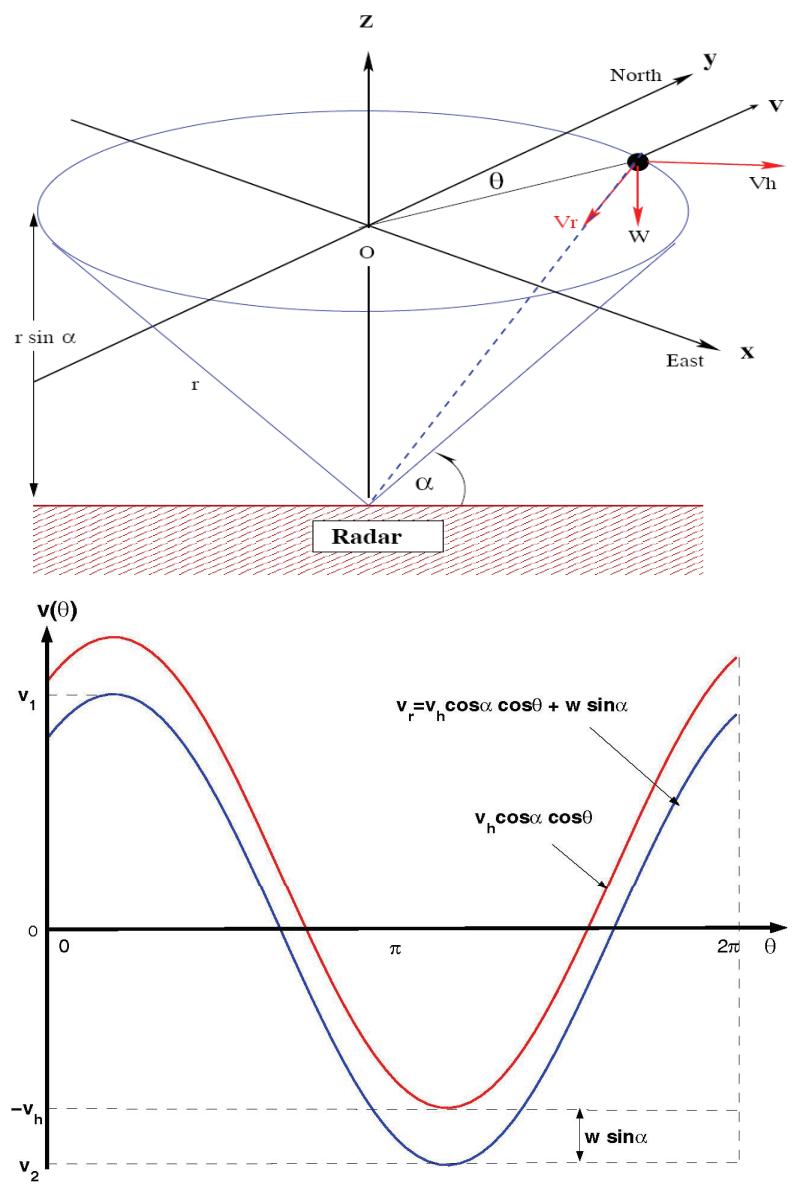

Figure 2. Geometry for scan of velocities on a Velocity Azimuth Display (VAD) circle (top) and the variation of the radial.

At each measurement point a Gaussian (or a modification of a Gaussian) distribution is introduced, the magnitude and spatial variation of which may represent the different error types shown in Figure 1. Examples of the type of artificial radial velocity field produced using Formula (3) are displayed in Figure 3 (top). Such data can be used to test the representation of the errors in the Doppler radial winds, and variational analysis schemes.

Air movement is 3-dimensional and varies over time and space. However, Doppler radar allows the measurement of only one (radial) component of the velocity of the targets at a specific range and azimuth. Since we only take the data from single radar in the present study rather than simultaneous measurements with three Doppler radars, we are forced to make a simplifying assumption to the structure of the observed wind field during the creation of Doppler products.

The simplest case is to consider a horizontally uniform wind field for both, horizontal and vertical (precipitation fall velocity) components. In such a case, if we make measurements of the velocity along circles centred at the radar by azimuthal scanning at a constant elevation angle
(PPI), we get, for a constant distance from the radar, a sinusoidal dependency of the measured radial velocity on the azimuthal angle. Assuming that the horizontal wind velocity $v_{h}$ and hydrometeor fall speed $w$ are uniform over the area being observed, then the mean Doppler velocity $v_{r}$ varied sinusoidally with maxima and minima occurring when the beam azimuth passes the upwind $(\theta$ $=0)$ and downwind $(\theta=\pi)$ directions, that is when

$$
\left.\begin{array}{l}
v=v \cos \alpha+w \sin \alpha, \text { when } \theta=0 \\
v=-v \cos \alpha+w \sin \alpha, \text { when } \theta=\pi
\end{array}\right\}
$$

Hence

$$
v_{h}=\frac{v_{r 1}-v_{r 2}}{2 \cos \theta}, \quad w=\frac{v_{r 1}+v_{r 2}}{2 \sin \theta}
$$

Then the horizontal divergence is given by the formula

$$
\operatorname{div} v_{h}=\frac{1}{\pi R \cos \theta} \int_{0}^{2 \pi} v_{r} d \theta-\frac{2 w \tan \theta}{R},
$$

where $R$ is the radius of the radar sampling circle at height $l$. However, Equation (6) is only valid for low elevation angles.

Using Formula (3) to derive the wind velocity, we compare a plot derived from Figure 3 (top) with a perfect sine wave displayed in Figure 3 (bottom). The impact of the simulated errors is to cause the differences between the data (dots) and the no error sine curve (solid line) shown. It is possible to use the simulator to investigate, in more detail, the impact of various errors on the radial winds. This is the subject of continuing study.

\section{3D-Var Data Assimilation}

3D-Var systems use an incremental formulation (for a review see, for example, [6]). Under the assumption that the background and observation errors are Gaussian, random and independent of each other, the optimal estimate of the Cartesian wind $X_{a}=X_{b}+\delta X$ in the analysis space is given by the incremental cost function,

$$
\begin{aligned}
J[\delta X]= & \frac{1}{2} \delta X^{T} B^{-1} \delta X+ \\
& \frac{1}{2}\left[H \delta X-Y+H X_{b}\right]^{T} E^{-1}\left[H \delta X-Y+H X_{b}\right],
\end{aligned}
$$

where $\delta X=X_{a}-X_{b}$ is the state vector of the analysis increments (the estimated radial winds is given by $\mathfrak{H} \delta X_{a}+\mathfrak{H} X_{b}, \quad X_{b}$ the state variable of the background Cartesian winds, and $Y$ denotes the observed radial winds in the observation space. $\mathfrak{H}$ is the nonlinear observation operator that relates the model variables to the observation variable and a transformation between the different grid meshes, and $H$ is the linear observation operator with elements $h=\partial \mathfrak{H} / \partial X$. Some constructions of the background and observation error covariance 
matrices $B$ and $E$ are given in [15]. Miller and Sun [16], and $\mathrm{Xu}$ and Gong [14] assumed that the observation error covariance matrix $E$ is diagonal with constant diagonal elements given by the estimated observation error, which was taken as $1 \mathrm{~m} / \mathrm{s}$ for typical radar observations. In the next Section, we provide a different approach to the representation of the error of observed radial winds.

To avoid the computationally overwhelming problem of inverting the covariance matrix $B$ in the minimization of the cost function (7), and to accelerate the convergence of the minimization algorithm, a pre-conditioning of the minimization problem is needed [15]. This can be achieved by defining a variable $U$ to be applied to the assimilation increment $\delta X \quad(U \delta X=\mathfrak{X})$ such that it transforms the forecast error $\varepsilon$ in the model space into $\tilde{\varepsilon}$ a variable of an identity covariance matrix (i.e., $<\tilde{\varepsilon}, \tilde{\varepsilon}^{T}>=\mathrm{I}$, where $<., .>$ is an inner product). This change of variable can be written as $\varepsilon=U^{-1} \tilde{\varepsilon}$. Thus

$$
B=<\varepsilon, \varepsilon>=U^{-1}<\tilde{\varepsilon}, \tilde{\varepsilon}^{T}>U^{-T} \text {, or } B^{-1}=U^{T} U .
$$
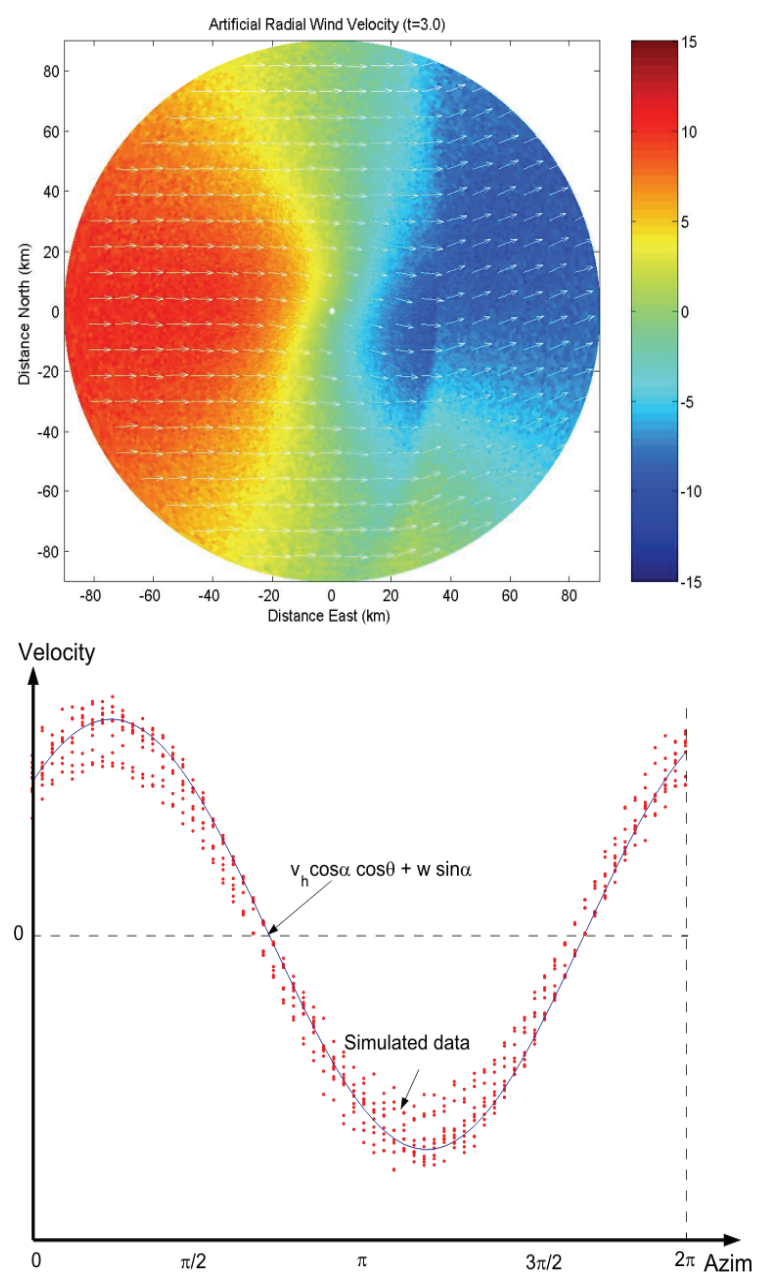

Figure 3. Artificial radial velocity with Gaussian noise.
This leads to a new representation of the incremental cost function of the form

$$
\begin{gathered}
J[\mathfrak{X}]=\frac{1}{2} \mathfrak{X}^{T} \mathfrak{X}+\frac{1}{2}\left[H U^{-1} \mathfrak{X}-Y+\mathfrak{H} X_{b}\right]^{T} E^{-1} \\
{\left[H U^{-1} \mathfrak{X}-Y+\mathfrak{H} X_{b}\right] .}
\end{gathered}
$$

With this cost function, no inversion of $B$ is needed. The control variables $\mathfrak{X}$ are velocity potential, stream function, unbalanced pressure and relative humidity. Here, we assume that the matrix $E$ includes the errors from the observations (original measurements), observation operator, and super-obbing procedure ${ }^{1}$. The 3D-Var analysis is then performed using continuous cycling procedure. The length of the assimilation window in each analysis is determined according to the model resolution. In each analysis cycle, the optimal analysis is obtained by minimizing the cost function (9) using iterative procedure.

The matrix $U^{-1}$ in (9) may be realized as

$$
U^{-1}=D F
$$

where $D$ is a diagonal matrix of standard deviation of the background error specified by the error estimation of numerical experiments, and $F$ is the square root of a matrix whose diagonal elements are equal to one, and offdiagonal elements are the background error correlation coefficients. In practical data assimilation for NWP, the full matrix $F$ is too large to compute explicitly or store into computer memory. Assumptions and approximations are made such that the effect of $F$ on the control variable $\mathfrak{X}$ in Equation (9) is achieved through the use of equivalent spatial filter. Following the work of Purser and McQuigg [17], Lorenc [18], and Hayden and Purser [19], the effect of $F$ using a recursive filter is defined by

$$
\left.\begin{array}{l}
\Upsilon_{i}=\alpha \Upsilon_{i-1}+(\alpha+1) X_{\mathrm{i}} \text { for } i=1,2, \ldots, n \\
\mathrm{Z}_{i}=\alpha \mathrm{Z}_{i-1}+(\alpha+1) Y_{\mathrm{i}} ; \text { for } i=n, n-1, \ldots, 1 ;
\end{array}\right\}
$$

where $X_{i}$ is the initial value at grid point $i, \Upsilon_{i}$ $(i=1, \ldots, n)$ is the value after filtering, and $Z_{i}$ is the initial value after one pass of the filter in each direction. $\alpha$ is the filter coefficients given in [15] by

$$
\alpha=1+\xi-\sqrt{\xi(\xi+2)}, \quad \xi=2 N \Delta x^{2} /\left(4 L^{2}\right)
$$

where $L$ is the horizontal correlation scale, $\Delta x$ is the grid spacing, and $N$ is the number of filter passes to be applied. This is a first-order recursive filter, applied in both directions to ensure zero phase change. Multipass filters ( $N$ greater than unity) are built up by repeating application of (11). This filter can be constructed in all

${ }^{1}$ Super-obbing procedure is a technique to combine (re-scale) the radar observations, using statistical interpolation, at a larger spatial scale which is compatible with the model. 
three directions. We next provide an approach to estimating the error covariance of radial velocity observations can be constructed in all three directions up by repeating application of (11). This filter can be constructed in all three directions.

We next provide some related issues due to nonlinearity of the dynamic system.

\section{Nonlinearity of the Atmospheric Dynamics}

In general, forecast skill increases not only by increasing model resolution, but also by improving the numerical models and the method of solution. However, even if we had a forecast model that represented atmosphere processes perfectly, we would never be able to predict the state of atmosphere accurately for long lead times. A chaotic behavior occurs (and leads to an unpredictable long-term evolution) when solving deterministic, nonlinear, dynamical systems that exhibit sensitivity to initial conditions. This occurs, because the nonlinear dynamical systems that describe the atmospheric behaviour are sensitive to small changes in initial conditions. In this section we consider, two issues associated with data assimilation, sensitivity analysis and biases due to nonlinearity.

Now, to what lead time forecasts remain skillful depends on how small errors in the initial conditions, boundary conditions, or model specifications grow to affect the state output or the forecast. Because errors tend to grow rapidly in processes that occur at smaller spatial-scales, then forecasts for small scale processes may be predictable only for few hours. However, forecasts of large scale processes can be predicted for perhaps two weeks ahead. Thus, when solving the forward problem, it is very important to assess the sensitivity of the state output variables of the dynamic system to small changes in the initial conditions. A knowledge of how the state variables can vary with respect to small changes in the initial data can yield insights into the behaviour of the model and assist the modelling process to determine (for example) the most sensitive area. The sensitivity analysis, of the dynamic system, entails finding the partial derivative of the state variable (or the analysis) with respect to the parameters, which is a big challenge in a large nonlinear system. For further study of sensitivity analysis due to the nonlinearity, we refer to see $[6,20,21]$.

It should also be noted that the predictability of the atmospheric state depends mainly on the accuracy of the parameter estimates (the control variables), when solving the inverse problem. Since the ultimate goal is to produce an analysis that gives the best forecast, it is desirable to have information about the effect on the analysis system (or the estimates) due to perturbing the observations (or noisy data), or small changes in the background;
See [6].

The nonlinear behavior of the wind field has been discussed in Lovejoy et al. [22]. Vertically propagating gravity waves are broken up by unstable layers (see for example Browning et al, 2009 [23]) which have fractal structures. Lovejoy et al. [22] show that one can readily make strongly nonlinear models based on localized turbulence fluxes which have wavelike unlocalized velocity fields, and this respecting the observed horizontal and vertical scaling. This turbulent anisotropic scaling can give rise to (nonlinear) dispersion relations not so different than those predicted by linear theory so it may be sufficient to reinterpret the empirical studies of waves in this anisotropic scaling framework.

We next provide an approach to estimating the error covariance of radial velocity observations.

\section{Errors in Observation Radial Winds}

In order to optimally assimilate Doppler radar radial velocity observations into NWP model, it is necessary to know their error covariances. We assume that the observational errors are uncorrelated in space and time. Under this assumption, the observation error covariance matrix $\Xi(\equiv E)$ in the cost function (9) can be reduced to a diagonal matrix. Then the matrix $E$, in Equation (9), is regarded as a weighting coefficient that reflects the relative precision of the data (measurement uncertainty and representativeness error). The matrix $\Xi$ can be expressed as:

$$
\Xi=\operatorname{diag}\left[\sigma^{2}(\epsilon)\right],
$$

where $\sigma^{2}(\in)$ is the error variance of the radial velocity $v_{r}$. The most common error in radar radial winds are 1) the noise in the radial velocity induced by the velocity gradient across the pulse volume with variance, $\bar{\sigma}^{2}\left(\in_{v}\right)$, and 2) the instrumental error due to hardware degradation of variance $\hat{\sigma}^{2}\left(\epsilon_{i}\right)$. Miller and Sun [16] state that these measurement errors need to be specified so that radar observations can be properly assimilated for NWP. However, they note that the mean radial velocity and spectral width estimators are proportional to the radar wavelength and the time spectral width [24], and therefore are rather impractical as estimates of the measurement errors. They therefore note a need for error estimators of radial velocity that can be obtained from the measurements themselves.

\subsection{Error Due to the Velocity Gradient}

The local sampling of the radial velocity is employed to approximate the error variance $\tilde{\sigma}_{v_{r}}^{2}$, since noisy data are usually associated with high values of radial velocity 
variance.

Errors in the original measurements of the radial velocity within each radar pulse volume depend on the strength of the returned signal and the spread (or width) of the Doppler velocity spectrum that depends on the velocity gradients. Since the radar scatterers in the pulse volume move randomly, we assume that the errors of the velocity gradient of the radar backscatterers are given by a normal (Gaussian) distribution, where,

$$
p d f\left(\epsilon_{v}\right)=\int_{-\infty}^{\infty} \frac{1}{\sqrt{2 \pi} \tilde{\sigma}} e^{-\epsilon_{v} / 2 \tilde{\sigma}^{2}} d \epsilon_{v} .
$$

The error variance $\tilde{\sigma}^{2}$ is modified by the velocity gradient (which varies with time) along the pulse volume. The variations of this velocity difference along the pulse volume cause the kinetic energy $(K E)$ of the moving scatterers to change. We will assume here for simplicity that this velocity difference is taken in the radial direction only. Here,

$$
\text { the rate of change in the } K E=\mathfrak{s} \Delta v \text {, }
$$

where $\mathfrak{I}$ is an arbitrary force applied to the scatterers and $\Delta v_{r}$ is the velocity difference along the pulse volume. Thus the increase in the kinetic energy in time interval $d t$ during which the scatterer moves a distance $d r$, is

$$
d(K E)=d W,
$$

where $W$ by is the work done the force $\mathfrak{I}$ the infinitesimal displacement $d r$ taken as the pulse length and over time $d t$.

Rogers and Trips [24] show that the change in the kinetic energy per unit mass can be expressed as

$$
d(K E)=\sigma^{2}\left(v_{r}\right)
$$

where $\sigma^{2}\left(v_{r}\right)$ is the variance of the mean Doppler velocity, which can be expressed by (see [24])

$$
\sigma^{2}\left(v_{r}\right)=\frac{\lambda}{8 \sqrt{\pi} M T} \varphi .
$$

Here $\lambda$ is the radar wavelength, $\varphi$ is the true spectral width, $M$ is the number of equally spaced pulses, and $T$ is the time between pulses. (The maximum unambiguous (Nyquist) velocity is $v_{n y q}=\lambda / 4 T$ ).

Nastom [25] investigated the factors impacting on the spectral width of Doppler radar measurements. For very small bandwidths it was found that the variance was dominated by the effects of wind speed changes along the radar beam. The expression for the variance was derived as a function of the beam elevation and the vertical wind shear. We therefore chose here to express the error variance, $\bar{\sigma}^{2}\left(\epsilon_{v}\right)$ of radial winds more simply in terms of the gradient variance along the pulse volume in a ra- dial direction as follows

$$
\bar{\sigma}^{2}\left(\epsilon_{v}\right)=\left(1-e^{-\left|\Delta v_{v} / v_{r}\right|}\right) \sigma^{2}\left(v_{r}\right),
$$

where $\Delta v_{r}$ is the gradient of the radial velocity, measured as a centred difference across the pulse volume. The error in radar radial winds due to the velocity gradient along the pulse volume varies with the range $R$. Figure 4 shows a proposed S-function for the observation errors as a function of the range, which is acceptable to represent the errors of the radial velocity. Note that as the range increases the error increases. This is to be expected as the radar beam gets wider and the pulse volume greater the kinetic energy variation of the scatters in the pulse volume increases, with increasing range.

\subsection{Error Due to Hardware Degradation}

Although the instrumental error can have a significant impact on the retrieval, in practice it is difficult to determine how this error varies with time. In this case we assume that the instrumental error does not vary temporally, and take the instrumental error variance as $\hat{\sigma}^{2}\left(\epsilon_{i}\right)$ assuming there is no hardware degradation with time. Therefore the total error variance of the radial winds is given by

$$
\hat{\sigma}^{2}(\in)=\breve{\sigma}^{2}\left(\epsilon_{v}\right)+\hat{\sigma}^{2}\left(\epsilon_{i}\right)
$$

at each $(r, \theta)$.

The instrumental error may not be a function of $(r, \theta)$, but is a function of time. In this case, we assume that this error is represented by a "skewed" distribution such as a Chi-Squared distribution with probability density function (for $v$ degrees of freedom) given by.

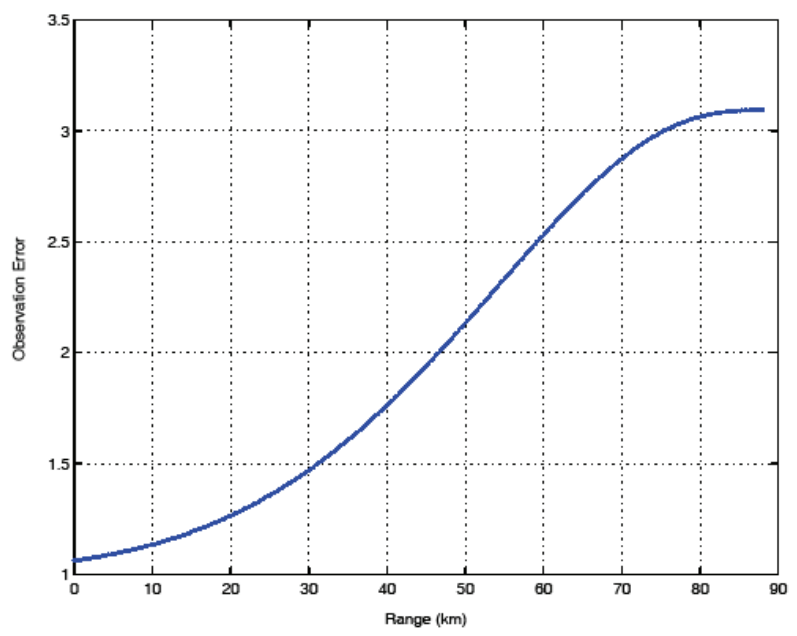

Figure 4. The proposed s-function for the observation errors as a function of the range. The variability of the error increases as the range. 


$$
p d f\left(\epsilon_{i}\right)=\frac{\epsilon^{(v-2) / 2} e^{-\epsilon_{i} / 2}}{2^{v / 2} \Gamma(v / 2)} .
$$

Here $\Gamma($.$) is the gamma function and \epsilon_{i}$ is the instrumental error. Thus the error variance of the degradation is defined by

$$
\hat{\sigma}^{2}\left(\epsilon_{i}\right)=\int p d f\left(\epsilon_{i}\right)\left(\epsilon_{i}-\bar{\epsilon}_{i}\right)^{2} d \epsilon_{i},
$$

where $\bar{\epsilon}_{i}$ is the mean of the instrumental errors.

\section{Direct Assimilation of PPI Data}

Due to the poor vertical resolution of radar data, a vertical interpolation of radar data from constant elevation levels to model Cartesian levels can result in large errors. For this reason a direct assimilation of PPI data with no vertical interpolation was recommended in $[1,7,8]$. However, radar data has better horizontal resolution than that of the model (the poorest polar radar data is approximately $0.5 \mathrm{~km}$ at the farthest range distance). An observation operator must be formulated to map the model variables from model grid into the observation locations such that the distance between the observations and model solution is estimated in the cost function. Thus, we take advantage of the vertical resolution of the model being much better than those of radar data. The observation operation, $\mathfrak{H}_{\mathfrak{e}}$, for mapping (and averaging) the data from the model vertical levels to the elevation angle levels is formulated as

$$
v_{\mathfrak{r}, \mathfrak{e}}=\mathfrak{H}_{\mathfrak{e}}\left(v_{r}\right)=\frac{\sum G v_{r} \Delta z}{\sum G \Delta z},
$$

where $v_{\mathfrak{r}, \mathfrak{e}}$ is the radial velocity on an elevation angle level, $v_{r}$ is the model radial velocity, and $\varnothing \mathrm{z}$ is the model vertical grid spacing. The function $G=e^{-\rho^{2} / \beta^{2}}$ represents the power gain of the radar beam, $\beta$ (in radians) is the beam half-width and $\rho$ is the distance from the centre of radar beam (in radians). The summation is over the model grid points that lie in a radar beam.

We next discuss the observation operator to convert the Cartesian model components to the radial components.

\subsection{Observation Operator}

There are two types of observation operators. One is used to interpolate and transfer the radar data from observation locations to the model grids. The second is used to map the model data into the observation locations. In the case of a direct assimilation of radar radial wind at constant elevation angles, which is not a model variable, the observation operator involves: 1) a bilinear interpola- tion of the NWP model horizontal and vertical wind components $u, v, w$ to the observation location; 2) a projection of the interpolated NWP model horizontal wind, at the point of measurement, towards the radar beam using the formula

$$
v_{h}=u \sin \theta+v \cos \theta,
$$

where $\theta$ is the the azimuth angle (clockwise from due North).

The elevation angle should include a correction which takes account of earth surface curvature and radar beam refraction (see [26]); then the third step 3) involves the projection of $v_{h}$ in the slantwise direction of the radar beam as

$$
\left.\begin{array}{c}
v_{r}=v_{h} \cos (\vartheta+\phi)+w \sin (\vartheta+\phi) \\
\phi=\tan ^{-1} \frac{r \cos (\vartheta)}{r \sin (\vartheta)+d+h}
\end{array}\right\} .
$$

Here $\vartheta$ is the elevation angle of the radar beam. The formula for $\phi$ represents approximately the curvature of the Earth. In the term $\phi, r$ is the range, $d$ is the radius of the Earth and $h$ is the height of the radar above the sea level; see [27].

\section{Pre-Processing of Doppler Radial Wind Data}

The major steps in processing the data before the assimilation are interpolation of data from/to a Cartesian grid, removing the noisy data, and filtering. Data quality control (QC) is a technique, which should be performed for each scan, to remove undesired radar echoes, such as ground clutter and anomalously propagated clutter (AP clutter), sea clutter, velocity folding, and noise using the threshold, that any velocity data with values less than, say, $0.25 \mathrm{~m} / \mathrm{s}$ and their corresponding reflectivity are removed. Unfolded Doppler velocity, in Doppler radar, is measured using both horizontally and vertically polarised pulses. This parameter is the component of the target velocity towards the radar (positive velocities are towards the radar). Noise are removed on the basis of the variance of the velocity at each pixel with its neighbours, which can occasionally remove good data with genuinely high variance; see [7].

\subsection{Super-Obbing Radial Wind Data}

Doppler radars produce raw radial wind data with high temporal and spatial density. The horizontal resolution of the data is around $300 \mathrm{~m}$ (that is too high to be used in the assimilation scheme) whereas the typical resolution of an operational mesoscale NWP model is of the order of several kilometers. To reduce the representativeness error, and correspond the observations to the horizontal 
model resolution, one may use spatial averages of the raw data, called super-observations. The desired resolution for the super-observations can be generated by defining parameters (which can be freely chosen) for the range spacing and the angle between the output azimuth gates.

As we have mentioned previously, a direct assimilation of PPI data with no vertical interpolation is recommended. Moreover assimilation using radar data directly at observation locations avoids interpolation from an irregular radar coordinate system to a regular Cartesian system, which can often be a source of error especially in the presence of data voids (see [6]). We have developed a software package, which is based on spatial interpolation in polar space, for processing of raw volume data of radial velocity in PPI format to super-obb the data to the required resolutions for the $3 \mathrm{D}-$ Var system in the Met Office (UK); see [1].

\section{Steps of NWP}

NWP is an initial-value problem

$$
\frac{\partial X}{\partial t}=F(X), \quad X\left(t_{0}\right)=X_{0}
$$

for which we should provide the initial conditions (ICs). These equations are in general "partial differential equations" of which the most important are equations of motion, the first law of thermodynamics, and the mass and humidity conservation equations. NWP can be summarized in the following three steps: The first is to collect all atmospheric observations for a given time. Second, those observations are diagnosed and analyzed (that represented in data assimilation) to produce a regular, coherent spatial representation of the atmosphere at that time. This analysis becomes the initial condition for time integration of NWP model that based on the governing differential equations of the atmosphere. Finally, these equations are solved numerically to predict the future states of the atmosphere. For further information about the governing equations of the evolution of the atmosphere, we refer to [28-30].

\section{Concluding Remarks}

The benefits of assimilating Doppler radar winds in NWP are that: 1) the Limited Area Models require observations with high spatial-temporal resolution to forecast the details of weather and its development; 2) Doppler radars are able to scan large volumes of the atmosphere, and provide high resolution measurements of reflectivity and radial velocity in forecasting of quickly developing mesoscale systems. We should mention here that the predictability of the atmospheric state depends mainly on the accuracy of the parameter estimates (the control variables), when solving the inverse problem. Since the ultimate goal is to produce an analysis that gives the best forecast. A particular challenge in the forecasting of the time evolution of atmospheric system is the nonlinearity of the system and the corresponding sensitivity of the initial conditions. The major impact of assimilating Doppler radial winds upon model performance is likely to arise from the impact of these data upon moist convective processes, through moisture related variables such as vertical velocity.

The aim of this paper was mainly to investigate issues concerned with the assimilation of Doppler radial winds into a NWP model using a 3D-Var system to improve the numerical forecasting in the Gulf Area. This is due to the fact that there is a witnessing rapid economical and technological development associated with considerable investments in infrastructure and developmental projects. The technique displayed in this paper can be applied in data collected in the Gulf area, especially in UAE: Both wind and visibility data inferred from the Radar measurements are the assimilated using 3D-Var system and MM5 mesoscale model are used to for the forecasts. The model experiments can be performed under different weather conditions, with particular emphasis on improving behavior of the model at the local scale and under high aerosol (dust/sand/pollution) conditions.

\section{Acknowledgement}

The authors thank Prof M. Maim Anwar for his valuable comments in this paper.

\section{References}

[1] F. A. Rihan, C. G. Collier, S. Ballard and S. Swarbrick, "Assimilation of Doppler Radial Winds into a 3D-Var System: Errors and Impact of Radial Velocities on the Variational Analysis and Model Forecasts," Quarterly Journal of the Royal Meteorological Society, Vol. 134, No. 636, 2008, pp. 1701-1716.

[2] C. G. Collier, Ed., "COST-75, Project, Advanced Weather Radar Systems 1993-1996," European Commission EUR 1954, Brussels, 2001, p. 362.

[3] R. Krzysztofowicz and C. G. Collier, Eds., "Quantitative Preceptation Forecasting II," Special Issue of Journal of Hydrology, Vol. 288, No. 1-2, 2004, pp. 225-236.

[4] S. G. Benjamin, D. Dévényi, S. Weygondt, K. J. Brundaye, J. M. Brown, G. A. Grell, D. Kim, B. E. Schwartz, T. G. Mirnova, T. L. Smith and G. S. Monikin, "An Hourly Assimilation Precsent Cycle: The RUC," Monthly Weather Review, Vol. 132, No. 11, 2004, pp. 494-518.

[5] M. Lindskog, H. Jarvinen, D. B. Michelson, "Development of Doppler Radar Wind Data Assimilation for the HIRLAM 3D-Var," COST-717, 2002.

[6] F. A. Rihan, C. G. Collier and I. Roulstone, "Four-Dimensional Variational Data Assimilation for Doppler 
Radar Wind Data," Journal of Computational and Applied Mathematics, Vol. 176, No. 1, 2005, pp. 15-34.

[7] J. Sun and N. A. Crook, "Dynamical and Physical Retrieval from Doppler Radar Observations Using a Cloud Model and its Adjoint. Part II: Retrieval Experiments of an Observed Florida Convective Storm," Journal of the Atmospheric Sciences, Vol. 55, No. 5, 1998, pp. 835-852.

[8] J. Sun and N. A. Crook, "Dynamical and Microphysical Retrieval from Doppler Radar Observations Using a Cloud Model and its Adjoint. Part I: Model Development and Simulated Data Experiments," Journal of the Atmospheric Sciences, Vol. 54, No. 12, 1997, pp. 1642-1661.

[9] J. D. Doviak and D. S. Zrnic, Doppler Radar Weather Observations, 2nd Edition, Academic Press, London/San Diego, 1993.

[10] J. Gong, L. Wang and Q. Xu, "A Three Step Dealiasing Method for Doppler Velocity Data Quality Control," Journal of Atmospheric and Oceanic Technology, Vol. 20, No. 12, 2003, pp 1738-1748.

[11] Y. Lin, P. S. Ray and K. W. Johnson, "Initialization for a Modeled Convective Storm Using Doppler Radar DataDerived Fields," Monthly Weather Review, Vol. 124, No. 1, 1993, pp. 2757-2775.

[12] P. T. May, T. Sato, M. Yamamoto, S. Kato, T. Tsuda and S. Fakao, "Errors in the Determination of Wind Speed by Doppler Radar," Journal of Atmospheric and Oceanic Technology, Vol. 6, No. 3, 1989, pp. 235-242.

[13] P. Saarikivi, "Simulation Model of a Signal Doppler Radar Velocity," University of Helsiriki, Department of Meteorology, Technology Report 4, 1987, p. 22.

[14] Q. Xu and J. Gong, "Background Error Covariance Functions for Doppler Radial Wind Analysis," Quarterly Journal of the Royal Meteorological Society, Vol. 129, No. 590, 2003, pp. 1703-1720.

[15] A. C. Lorenc, "Development of an Operational Variational Scheme," Journal of the Meteorological Society of Japan, Vol. 75, No. 2, 1997, pp 339-346.

[16] L. J. Miller and J. Sun, "Initialization and Forecasting of Thrundstorms: Specification of Radar Measurement Errors," 31th Conference on Radar Meteorology, American Meteorological Society, Seattle, Washington, 2003, pp. 146-149.

[17] R. J. Purser and R. McQuigg, "A Successive Correction Analysis Scheme Using Numerical Filter," Met Office Technical Note, Vol. 154, 1982, p. 17.
[18] A. C. Lorenc, "Iterative Analysis Using Covariance Functions and Filters," Quarterly Journal of the Royal Meteorological Society, Vol. 118, No. 505, 1992, pp. 569-591.

[19] C. M. Hayden and R. J. Purser, "Recursive Filter for Objective Analysis of Meteorological Fields: Applications to NESDIS Operational Processing," Journal of Applied Meteorology, Vol. 34, No. 1, 1995, pp. 3-15.

[20] D. G. Cacuci, "Sensitivity Theory for Nonlinear Systems, I: Nonlinear Functional Analysis Approach," Journal of Mathematical Physics, Vol. 22, No. 12, 1981, pp. 27942802.

[21] D. G. Cacuci, "Sensitivity Theory for Nonlinear Systems, II: Extensions to Additional Classes of Response," Journal of Mathematical Physics, Vol. 22. No. 12, 1981, pp 2803-2812.

[22] S. Lovejoy, A. F. Tuck, S. J. Hovde and D. Schertzer, "Do Stable Atmospheric Layers Exist?" Geophysical Research Letters, Vol. 35, No. 1, 2008, pp. 1-4.

[23] K. A. Browning, J. H. Marsham, A. M. Blyth, S. D. Mobbs, J. C. Nochol, M. Perry and B. A. Wite, "Observations of Dual Slantwise Circulations above a Cool Undercurrent in a Mesocale Convective Storm," Quarterly Journal of the Royal Meteorological Society, Vol. 136, No. 647, 2009, pp. 354-373.

[24] R. J. Doviak and D. S. Zrnic, Doppler Radar Weather Observations, 2nd Edition, Academic Press, London/San Diego, 1993.

[25] G. D. Nastrom, "Doppler Radar Spectral Width Broadening Due to Beamwidth and Wind Shear," Annales Geophysicae, Vol. 15, No. 6, 1997, pp 786-796.

[26] M. Lindskog, M. Gustafsson, B. Navascuès, K. S. Mogensen, X.-Y. Huang, X. Yang, U. Andrae, L. Berre, Thorsteinsson and J. Rantakokko, "Three-Dimensional Variational Data Assimilation for a Limited Area Model, Part II: Observation Handling and Assimilation Experiments," Tellus A, Vol. 53, No. 4, 2001, pp. 447-468.

[27] K. Salonen, "Observation Operator for Doppler Radar Radial Winds in HIRLAM 3D-Var," Proceedings of ERAD, 2002, pp. 405-408.

[28] R. Daley, Atmospheric Data Analysis, Cambridge University Press, Cambridge, 1996.

[29] I. N. Jame, Introduction to Circulating Atmospheres, Cambidge University Press, Cambridge, 1994.

[30] E. Kalnay, Atmospheric Modeling, Data Assimilation and Prectipility, Cambrige Press, Cambridge, 2003. 